\section{$\underset{\substack{\text { hommes } \\ \text { \& migrations }}}{ }$}

\section{Hommes \& migrations}

Revue française de référence sur les dynamiques

migratoires

$1317-1318 \mid 2017$

L'Europe en mouvement

\title{
Prix littéraire de la Porte Dorée : la sélection 2017
}

\section{Nihad Jnaid}

\section{OpenEdition \\ Journals}

\section{Édition électronique}

URL : http://journals.openedition.org/hommesmigrations/3928

DOI : 10.4000/hommesmigrations.3928

ISSN : 2262-3353

\section{Éditeur}

Musée national de l'histoire de l'immigration

\section{Édition imprimée}

Date de publication : 1 avril 2017

Pagination : 203-206

ISBN : 978-2-919040-38-4

ISSN : $1142-852 X$

\section{Référence électronique}

Nihad Jnaid, «Prix littéraire de la Porte Dorée : la sélection 2017 », Hommes \& migrations [En ligne], 1317-1318 | 2017, mis en ligne le 01 avril 2017, consulté le 14 septembre 2020. URL : http:// journals.openedition.org/hommesmigrations/3928 


\section{PRIX LITTÉRAIRE DE LA PORTE DORÉE : LA SÉLECTION 2017}

Par NIHAD JNAID, responsable du Prix littéraire de la Porte Dorée

Le comité de lecture de l'Établissement public du Palais de la Porte Dorée vient d'annoncer la sélection de la 8 édition du Prix littéraire de la Porte Dorée dont la remise aura lieu le 8 juin prochain. Entre les coups de cœur des uns et les préférences des autres, six titres ont été retenus. Cette édition se distingue par une forte présence féminine, quatre auteures sur six, et par la sélection de trois premiers romans.

\section{LES MEMBRES DU JURY 2017}

Doan BUI, journaliste, présidente du jury, lauréate 2016 pour son roman Le silence de mon père (L'Iconoclaste).

Judith ROZE, directrice du Département Langue française, Livre et Savoirs Institut français.

Alexis NUSELOVICI, Professeur, responsable de la Chaire "Exil et migrations", Collège d'études mondiales (FMSH).

Yvan AMAR, journaliste RFI.

Mustapha HARZOUNE, critique littéraire.

Elena GURITANU, libraire.

Maryse MONDAIN, libraire.

Délégué de la classe de $2^{\text {de }} \mathrm{D}$ du lycée Corot de Savigny-sur-Orge.

Délégué de la classe de $1^{\text {ère }}$ ES - L du lycée Galilée de Cergy. 
LIVRES
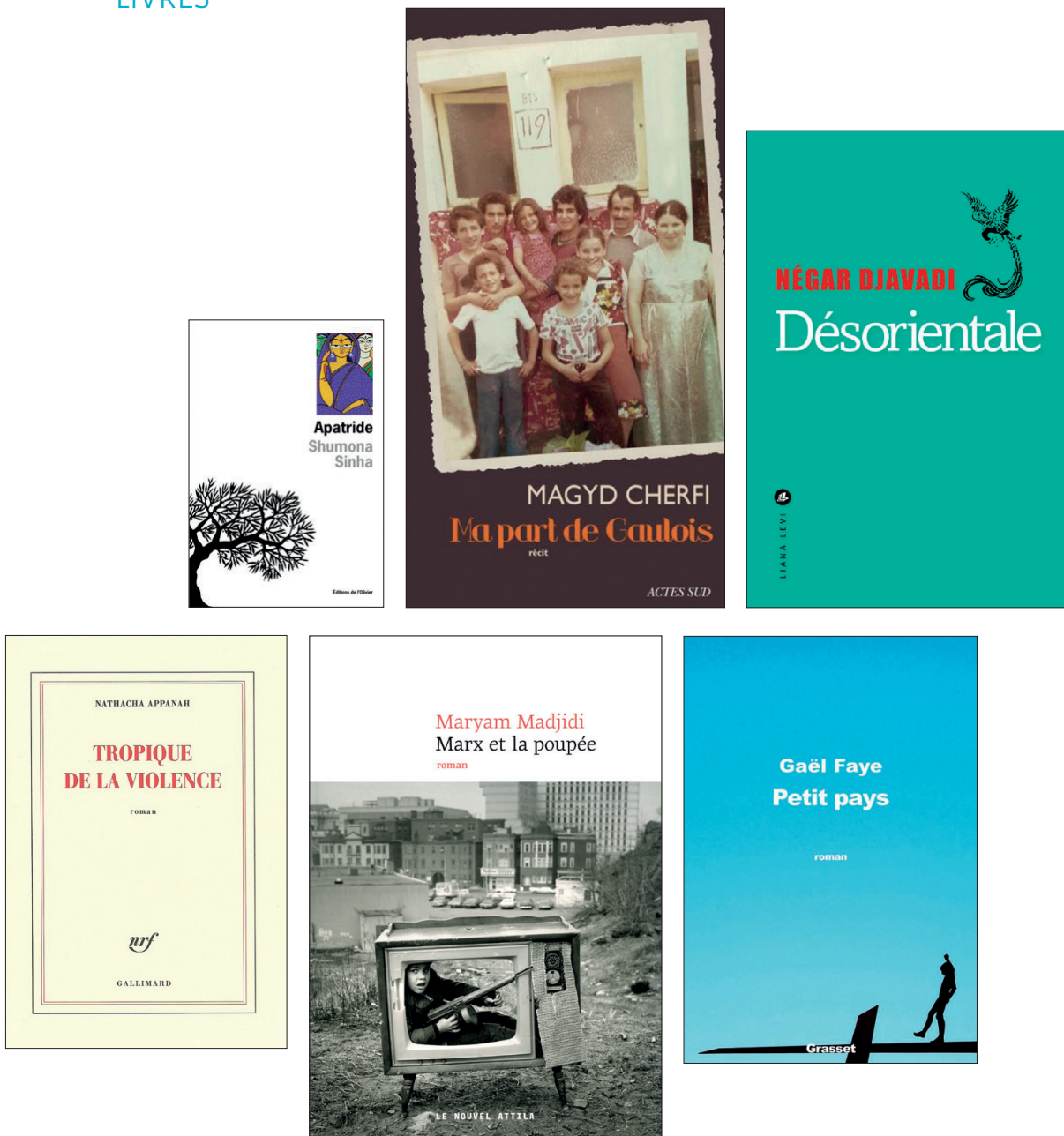

I es thèmes abordés dans la sélection font aussi la part belle aux récits à hauteur d'enfant. En effet, les souvenirs d'enfance révélés dans Petit pays, l'arrivée en France et l'enracinement de la narratrice de Marx et la poupée, de Kimiâ dans Désorientale et surtout l'enfance malheureuse de Moïse dans Tropique de la violence offrent un riche panel de récits d'enfants.

La sélection invite à voyager dans l'histoire et la géopolitique contemporaines. Qu'il s'agisse de l'histoire politique de l'Iran, de l'arrivée au pouvoir de Mitterrand, de la guerre fratricide au Rwanda et de ses répercussions sur le Burundi voisin, de la situation sociale des femmes en Inde ou des tensions à Mayotte, les six titres de cette sélection nous révèlent un monde en tension, fait de violences. Ces romans porteurs d'espoirs enchantent le quotidien. La littérature est cette voix qui permet, à travers ces œuvres de fiction ou autobiographiques, de découvrir l'autre, de se rapprocher de lui et de s'évader, séduit par les richesses culturelles et linguistiques qui traversent cette édition. 


\section{Nathacha Appanah}

Tropiques de la violence

Gallimard

Sur l'île de Mayotte affluent les migrants en provenance des Comores. Les enfants perdus font la loi. Le roman suit cing destins qui se croisent, cing voix, vivantes ou mortes, qui racontent la violence et la dure réalité des habitants de cette île. Moïse, l'« enfant du djinn », un œil noir, l'autre vert, recueilli par Marie, l'infirmière blanche, est le personnage pivot du roman. Mais la vie les sépare et Moïse va connaître l'enfer des enfants de la rue et d'une jeunesse livrée à elle-même dans un bidonville appelé « Gaza ». La quête de ses origines, mènera "Mo » du côté de Bruce, le chef de Gaza, de Stéphane, le militant d'une ONG, et d'Olivier, un policier qui tente de le protéger. À travers les histoires de Marie, Moïse, Bruce, Olivier et Stéphane, Nathacha Appanah décrit Mayotte comme une île à la fois « magnifique et terrible».

\section{Magyd Cherfi}

Ma part de Gaulois

Actes Sud

C'est l'année du baccalauréat pour Magyd, petit Beur de la rue Raphaël, quartier nord de Toulouse. Une formalité pour les Français, un événement pour l'« indigène » et surtout pour sa mère. Le premier bac de la cité où avoir ce diplôme rime avec "intello ». Dans cette cité, Samir, le militant, Momo, l'artiste de la tchatche, et Magyd font l'expérience du "vivre ensemble ». Entre soutien scolaire aux plus jeunes et soutien moral aux filles malmenées, une génération joue les grands frères et les ambassadeurs entre familles et société, tout en se cherchant des perspectives d'avenir exaltantes. Avec les rumeurs accompagnant l'arrivée au pouvoir de Mitterrand, Ma part de Gaulois raconte l'intégration, la construction identitaire et le rêve républicain de toute une jeunesse issue de l'immigration. Cherfi résume son roman ainsi : " $L a$ plume m’a séparé de mes compagnons d'infortune, tous ces "Mohamed" de ma banlieue nord hachés menus par une société qui a rêvé d'un "vivre ensemble" sans en payer le prix. Je raconte une fêlure identitaire, un rendez-vous manqué... Pourtant la rencontre de la France et de sa banlieue n’a pas eu lieu, elle n’a toujours pas vu la lumière car l'exception française persiste, celle d'être français et de devoir le devenir... "

\section{Négar Djavadi}

\section{Désorientale}

Liana Levi

Kimiâ suit un protocole d'insémination artificielle pour avoir un enfant avec son amie Anna. La nuit, elle mixe du rock alternatif. Deux aspects de sa "désorientalisation " s'entremêlent avec les souvenirs de son pays natal, l'Iran. $\mathrm{Au}$ fil des flash-back et des souvenirs, le lecteur découvre la saga familiale des Sadr et toute une jeunesse déracinée et marquée par l'histoire politique de I'Iran. Kimiâ convoque trois générations et s'adresse au lecteur, comme à un témoin de l'évolution des événements et des personnages, pour parler de ses grands-parents, de ses parents, Darius et Sara, éternels opposants au régime en place ; celui du Shah jusqu'en 1979, puis celui de Khomeiny. Dans Désorientale, on croise également les oncles de Kimiâ, 
numérotés de 1 à 6 pour tenir l'attention du lecteur jusqu'au bout, mais aussi, et entre autres, Siouxie, Woody Allen, Michel Foucault et des punks bruxellois. Ce roman nous emmène en voyage à travers l'Arménie, I'Iran, Berlin, Bruxelles, Istanbul, Paris et même Deir-Ezzor en Syrie.

\section{Gaël Faye}

Petit pays

Grasset

"Je ne sais vraiment pas comment cette histoire a commencé " prévient le narrateur dès la première page. Nourri de "réminiscences d'enfance ", ce roman raconte l'histoire de Gabriel. Un garçon de 10 ans qui vit dans un quartier résidentiel de Bujumbura, une impasse protégée où on grandit parmi "des gens de maison ", où vivait une classe moyenne privilégiée, couples mixtes, diplomates africains, vieux expatriés, et où on va au Lycée français. La guerre vient mettre fin à cet univers paisible et bouleverse l'enfance heureuse de Gabriel. C'est plus précisément le récit des deux années 1992 et 1994 pendant lesquelles le garçon, entre ses 10 et 12 ans, voit basculer son existence. De sa hauteur d'enfant, Gabriel s'interroge sur les " silences et les non-dits des uns, les sous-entendus et les prédictions des autres. Il y avait des fractures invisibles, des soupirs, des regards que je ne comprenais pas ». Petit pays raconte la guerre mais aussi la découverte des identités ethniques, des assassinats, des déplacements et des conflits qui divisent la ville en «quartiers hutu » et « quartiers tutsi ». Puis il y a la blessure de l'exil, celle de la séparation, les réfugiés et le génocide. "Le génocide est une marée noire, ceux qui ne s'y sont pas noyés sont mazoutés à vie.»

\section{Maryam Madjidi}

Marx et la poupée

Le nouvel Attila

Maryam a six ans lorsqu'elle arrive à Paris. Le roman est divisé en trois parties autour de trois naissances, hormis la première, ces naissances marquent une étape d'émancipation dans la vie de cette jeune femme qui passe du rejet total de sa langue maternelle et du refus d'adopter le français à un état de réconciliation entre les deux langues. Il est donc question de langues mais aussi de cultures, de poésie. Les poèmes d'Omar Khayyam ponctuent les scènes de rencontres drôles et authentiques. Comment trouver sa place en France, comment accepter d'être multiple et riche de sa diversité ? C'est le roman d'un aller-retour France-Iran à vie.

\section{Shumona Sinha}

\section{Apatride}

Éditions de l'Olivier

Esha vit à Paris, la ville où elle rêvait de s'installer avant de quitter l'Inde. Une vie de femme immigrée qui tente, seule, de cons-truire une existence "normale ". Des papiers en règle, un logement, enseignante... mais la difficulté de trouver sa place persiste. L'exil qui semblait la seule solution pour changer de vie se révèle une rupture violente. Mina, fille de paysans amoureuse de son cousin, vit près de Calcutta. Elle est pauvre et tente aussi de trouver sa place. Deux femmes, deux exils et une violence commune. 